\title{
Satisfacción del familiar del paciente en la Unidad de Cuidados Intensivos del Hospital Nacional Dos de Mayo, 2018
}

\author{
Satisfaction of the patient's family member in the Intensive Care Unit of the Hospital \\ Nacional Dos de Mayo, 2018
}

\author{
Andrea Canchero-Ramirez ${ }^{1, a}$, Juan P. Matzumura-Kasano ${ }^{2, b}$, Hugo Gutiérrez-Crespo $2, c$ \\ ${ }^{1}$ Servicio de Cuidados Intensivos, Hospital Nacional Dos de Mayo. Lima, Perú. \\ ${ }^{2}$ Facultad de Medicina, Universidad Nacional Mayor de San Marcos. Lima, Perú. \\ ${ }^{a}$ Enfermera, magister en gerencia de servicios de salud. ORCID: http://orcid.org/0000-0003-2790-8230 \\ ${ }^{b}$ Médico gineco obstetra, doctor en medicina, Vicedecano de Investigación y Posgrado. ORCID: http://orcid.org/0000-0003-0231-0187 \\ 'Obstetra, magister en docencia e investigación en salud. ORCID: http://orcid.org/0000-0003-1097-6990
}

An Fac med. 2019;80(2):177-82 / DOI: https://10.15381/anales.802.16412

\begin{abstract}
Correspondencia:
Andrea Canchero Ramirez

andreacr14@hotmail.com
\end{abstract}

Recibido: 20 de marzo 2019

Aceptado: 16 de mayo 2019

Publicación en línea: 28 de junio 2019

Conflictos de interés: Los autores declaran no tener conflictos de interés

Fuente de financiamiento:

Autofinanciado

Citar como: Canchero-Ramirez A, Matzumura-Kasano J, Gutiérrez-Crespo H. Satisfacción del familiar del paciente en la Unidad de Cuidados Intensivos del Hospital Nacional Dos de Mayo, 2018. An Fac med. 2019;80(2):177-82. DOl: https:// 10.15381/anales.802.16412

\section{Resumen}

Introducción. La unidad de cuidados intensivos es un área destinada al cuidado y tratamiento para salvar y preservar la vida. Objetivo. Determinar la satisfacción del familiar del paciente en una unidad de cuidados intensivos. Métodos. Estudio de diseño no experimental de corte transversal. La muestra estuvo conformada por familiares de pacientes hospitalizados en la Unidad de Cuidados Intensivos del Hospital Nacional Dos de Mayo. Se utilizó el instrumento The Patient Satisfaction Questionnaire Short Form diseñado por Marshall GN. y Hayds RD. Resultados. Se entrevistaron 127 familiares; $54 \%$ de los pacientes correspondieron al género masculino, $87,4 \%$ tenían Seguro Integral de Salud y $92,1 \%$ ingresó por primera vez a la unidad. El equipamiento para la atención obtuvo puntuación favorable, la atención personalizada fue desfavorable. La dimensión forma interpersonal obtuvo $60,5 \%$ de satisfacción y la dimensión satisfacción general $79,9 \%$ de satisfacción. Conclusiones. Todas las dimensiones superaron el $60 \%$ de satisfacción y la puntuación total correspondió a 70,5\% de satisfacción.

Palabras clave: Familia; Pacientes; Unidad de Cuidados Intensivos

\section{Abstract}

Introduction. The intensive care unit is an area dedicated to care and treatment to save and preserve life. Objective. Determine the satisfaction of the patient's family member in the intensive care unit. Methods. Non-experimental cross-sectional design study. The sample consisted of the patient's family member hospitalized in the Intensive Care Unit of the National Hospital Dos de Mayo. The instrument was used The Patient Satisfaction Questionnaire Short Form designed by Marshall GN and Hayds RD. Results. 127 relatives were interviewed; $54 \%$ of the patients corresponded to the male gender, $87,4 \%$ had Comprehensive Health Insurance and 92,1\% entered the unit for the first time. The equipment for the attention obtained favorable score, the personalized attention was unfavorable. The interpersonal form dimension obtained $60,5 \%$ satisfaction and the general satisfaction dimension $79,9 \%$. Conclusions. All dimensions exceeded $60 \%$ satisfaction and the total score corresponded to $70,5 \%$ satisfaction.

Keywords: Family; Patients; Intensive Care Unit 


\section{INTRODUCCIÓN}

Las unidades de cuidados intensivos son ambientes complejos y multidisciplinarios donde trabajan muchos profesionales a todos los niveles y en un contexto de estrés, por lo que muchas veces los aspectos sociales y psicológicos de los familiares pueden pasar a un segundo plano $^{1,2,3}$. Estos ambientes proporcionan un entorno de alta tecnología centrado en el cuidado y tratamiento óptimo para salvar y preservar la vida del paciente ${ }^{4}$. Por lo general, las unidades de cuidados intensivos suelen ser lugares donde la tecnología y la efectividad práctica predominan sobre las relaciones personales y la calidad humana. El ingreso de un paciente provoca una desestabilización total del núcleo familiar desde el punto de vista psicológico y emocional ${ }^{5}$.

Es importante enfatizar que la familia es quien acompaña la evolución del paciente, de tal manera que tiene la necesidad de recibir información completa, honesta y oportuna, que tengan las facilidades y acceso para visitar al paciente cuando lo desee, asegurándose de que reciba un trato cálido y humano, teniendo en cuenta la seguridad del paciente en su totalidad ${ }^{6}$. Los miembros de la familia de un paciente crítico, cumplen un rol importante y difícil como representantes sustitutos para la toma de decisiones, ya que el paciente no lo puede realizar por el estado de salud en que se encuentra; así, en muchas ocasiones presentan estrés significativo, carga emocional y económica?

Los profesionales de salud tienen el compromiso y el deber de aclarar las dudas de los familiares ya que diversos factores pueden interferir en la satisfacción por la atención recibida en la unidad de cuidados intensivos. Por ello, el equipo de salud tiene diferentes herramientas de atención para contribuir al conocimiento y la evaluación ${ }^{8,9}$. Teniendo en cuenta que la satisfacción es el cumplimiento de las necesidades o requisitos fundamentales que, al satisfacer, alivia o disminuye la angustia de los miembros de la familia; mientras que la satisfacción del familiar con respecto a la atención refleja el grado en que los profesionales de la salud satisfacen las necesidades y expectativas percibidas por los miembros de la familia ${ }^{10}$.
La evaluación de las necesidades y el grado de satisfacción de los miembros de la familia de los pacientes de la unidad de cuidados intensivos, se convierten en una parte esencial de la atención de los profesionales de la salud, quienes tienen el compromiso con la atención, reduciendo el dolor y el sufrimiento de las personas que tienen un miembro de la familia en estado crítico ${ }^{11}$. Las familias asocian la satisfacción con la información clara, lo que les permite comprender las necesidades de atención de sus miembros y la actitud del equipo médico. Por lo tanto, la implementación de medidas de comodidad en la sala de espera con un ambiente armonioso limpio y agradable puede contribuir a la satisfacción de la familia ${ }^{9}$. Un estudio realizado por Stricker y col., reportó que la buena comunicación con la familia de los pacientes hospitalizados en la unidad de cuidados intensivos, disminuye la estancia hospitalaria. También se demostró que la comunicación efectiva mejora la satisfacción familiar y el bienestar psicológico ${ }^{11}$.

En la actualidad, existen diversas herramientas, en su mayoría cuestionarios, que miden la satisfacción de la familia mediante la obtención de datos, los mismos que deben ser precisos y con demostrada validez y confiabilidad, ya que son elementos esenciales para el desarrollo de una investigación científica ${ }^{12.13}$. La revisión ha evidenciado un vacio en la producción de investigaciones que describan la satisfacción de los familiares en el caso de pacientes que permanecen en un área critica. Por ello, el objetivo del presente estudio busca determinar la satisfacción del familiar del paciente hospitalizado en la Unidad de Cuidados Intensivos del Hospital Nacional Dos de Mayo durante el año 2018.

\section{MÉTODOS}

Se realizó el estudio mediante un diseño no experimental, tipo descriptivo de corte transversal y la población de estudio estuvo conformada por los familiares directos de los pacientes que ingresaron a la unidad de cuidados intensivos con una permanencia $\geq$ a 72 horas, durante los meses de agosto a noviembre del año 2018; se excluyeron a los pacientes fallecidos, familiares menores de edad y a aquellos que no desearon participan de manera voluntaria. Por la naturaleza del ámbito del estudio, se consideró utilizar una muestra no probabilística por conveniencia.

La técnica empleada consistió en la encuesta y se utilizó el instrumento The Patient Satisfaction Questionnaire Short Form (PSQ-18), diseñado por Marshall GN y Hays $R^{14}$, de aplicación en el ámbito de las unidades de cuidados intensivos y contiene 18 preguntas, divido en siete dimensiones; las respuestas de cada pregunta están conformadas mediante una escala de Likert, que van desde 1 (totalmente en desacuerdo) hasta 5 (totalmente de acuerdo). Cabe señalar que las preguntas con enunciado afirmativo se modificaron con una codificación inversa, según se detalla en la tabla 1. Para la de-

Tabla 1. Distribución y puntuación inversa de las preguntas de la encuesta The Patient Satisfaction Questionnaire Short Form (PSQ-18) aplicada a familiares de pacientes hospitalizados en la Unidad de Cuidados Intensivos del Hospital Dos de Mayo, 2018.

\begin{tabular}{lcc} 
Items & Valores originales & Valores \\
& 1 & 5 \\
$1,2,3,5,6,11,15,18$ & 2 & 4 \\
& 3 & 3 \\
& 4 & 2 \\
$4,7,10,12,13,14,16,17$ & 5 & 1 \\
\hline & 1 & 1 \\
& 3 & 2 \\
& 4 & 3 \\
& 5 & 4 \\
\hline
\end{tabular}

Fuente. Adaptado por Chakraborty y col. ${ }^{12}$. 
terminación de la satisfacción expresada en porcentajes, se tomaron en cuenta las recomendaciones de Chakraborty y col. ${ }^{16}$, mediante la siguiente secuencia: se registró la valoración del puntaje obtenido por cada dimensión, se identificó el puntaje máximo posible; luego, se dividió el puntaje obtenido entre el puntaje máximo y se multiplicó por la constante 100, tal como se detalla en la tabla 2. La confiabilidad según el coeficiente de alfa de Cronbach por dimensiones estuvo contemplada de la siguiente manera: satisfacción general $(0,9)$, calidad técnica $(0,9)$, manera interpersonal $(0,8)$, comunicación $(0,9)$, aspectos financieros $(0,9)$, tiempo que pasa con el médico $(1)$, accesibilidad-conveniencia $(0,9)$ y confiabilidad total $(0,9)$.

Los datos se presentan como media \pm desviación estándar, se realizó una conversión del formato de respuestas a una escala numérica expresada en porcentajes de satisfacción, en donde el valor de 0 es el peor de los resultados y 100 es el mejor. Los cálculos se realizaron con el programa SPSS versión 21 en español. El estudio fue aprobado por la Oficina de Investigación y Docencia así como por el Comité de Ética del Hospital Nacional Dos de Mayo. A todos los familiares entrevistados se les solicitó consentimiento informado previo a la aplicación de la encuesta, asegurándoles confidencialidad.

Tabla 2. Metodología para el cálculo de satisfacción por dimensiones, de la encuesta The Patient Satisfaction Questionnaire Short Form (PSQ-18) aplicada a familiares de pacientes hospitalizados en la Unidad de Cuidados Intensivos del Hospital Dos de Mayo, 2018.

\begin{tabular}{lcccc} 
Dimensión & Ítems & $\begin{array}{c}\text { Puntaje } \\
\text { máximo } \\
\text { posible }\end{array}$ & Media máxima & $\begin{array}{c}\text { Nivel de } \\
\text { satisfacción en } \\
\text { porcentaje }\end{array}$ \\
\hline Satisfacción general & $3+17$ (A) & 10 & 5 & $\mathrm{~A} / 10100$ \\
\hline Calidad técnica & $2+4+6+14(\mathrm{~B})$ & 20 & 5 & $\mathrm{~B} / 20100$ \\
\hline Forma interpersonal & $10+11(\mathrm{C})$ & 10 & 5 & $\mathrm{C} / 10100$ \\
\hline Comunicación & $1+13(\mathrm{D})$ & 10 & 5 & $\mathrm{D} / 10100$ \\
\hline Aspectos financieros & $5+7(\mathrm{E})$ & 10 & 5 & $\mathrm{E} / 10100$ \\
\hline $\begin{array}{l}\text { Tiempo dedicado por } \\
\text { médico }\end{array}$ & $12+15(\mathrm{~F})$ & 10 & 5 & $\mathrm{~F} / 10100$ \\
\hline $\begin{array}{l}\text { Accesibilidad y } \\
\text { conveniencia }\end{array}$ & $8+9+16+18(\mathrm{G})$ & 20 & 5 & $\mathrm{G} / 20100$ \\
\hline $\begin{array}{l}\text { Puntuación total } \\
\text { Puntas }\end{array}$ & 18 preguntas & 90 & 5 & $\mathrm{PT} / 90100$
\end{tabular}

Fuente. Adaptado por Chakraborty y col. ${ }^{12}$.

\section{RESULTADOS}

Se entrevistó a 127 familiares de los pacientes que ingresaron a la unidad de cuidados intensivos; la edad promedio correspondió a 49,69 \pm 16; 54\% fueron hombres. Con respecto al grado de instrucción 59,8\% refirieron tener estudios secundarios y $16,5 \%$ estudios superiores. $56,6 \%$ de los pacientes tuvieron una estancia hospitalaria entre de 1 a 10 días. En 51,9\% de los pacientes el motivo de ingreso fue médico; $87,4 \%$ de los pacientes pertenecieron al Seguro Integral de Salud. Finalmente, 92,1\% de los pacientes ingresaron por primera vez a la unidad. Tabla 3.

En la dimensión satisfacción general se observó que ambas preguntas obtuvieron una media de 3,94. En la dimensión calidad técnica, con respecto al enunciado "área y equipamiento necesario para la asistencia médica", se obtuvo una media de 4,02 $\pm 0,63$. En el enunciado "dudas del familiar sobre la capacidad médica", se obtuvo una media de 3,77 $\pm 0,94$. En los resultados de la dimensión forma interpersonal, correspondiente al indicador "los médicos brindan atención personalizada", se obtuvo una media de $2,1 \pm 0,64$. Respecto a la dimensión comunicación, el enunciado, "claridad en la explicación del médico con los familiares" reflejó una media de 3,99 $\pm 0,61$. En la dimensión aspectos financieros respecto al enunciado "gasto excesivo que supe- 
Tabla 4. Resultados de los enunciados por dimensiones de la encuesta The Patient Satisfaction Questionnaire Short Form (PSQ-18) aplicada a familiares de pacientes hospitalizados en la Unidad de Cuidados Intensivos del Hospital Dos de Mayo, 2018.

Dimensión satisfacción general

\begin{tabular}{|c|c|c|}
\hline La atención médica que recibe mi familiar es adecuado & 3,94 & 0,66 \\
\hline Estoy insatisfecho con algunas cosas sobre la atención médica que recibe mi familiar & 3,94 & 0,86 \\
\hline \multicolumn{3}{|l|}{ Dimensión calidad técnica } \\
\hline Cree que el área donde se encuentra su familiar tiene todo lo necesario para proporcionales asistencia médica & 4,02 & 0,63 \\
\hline A veces me pregunto si su diagnóstico y tratamiento médico son correctos & 3,54 & 1,06 \\
\hline Cuando brindan asistencia médica a mi familiar, tienen cuidado de verificar todo & 3,65 & 0,74 \\
\hline Tengo algunas dudas sobre la capacidad de los médicos que tratan a mi familiar & 3,77 & 0,94 \\
\hline \multicolumn{3}{|l|}{ Dimensión forma interpersonal } \\
\hline Los médicos actúan profesionalmente brindándome una atención personalizada & 2,10 & 0,64 \\
\hline Los médicos me tratan de una manera amable y cortés & 3,94 & 0,67 \\
\hline \multicolumn{3}{|l|}{ Dimensión comunicación } \\
\hline Los médicos son claros en explicar el motivo de los exámenes médicos & 3,99 & 0,61 \\
\hline Los médicos a veces ignoran lo que les digo & 3,87 & 0,93 \\
\hline \multicolumn{3}{|l|}{ Dimensión aspectos financieros } \\
\hline Estoy seguro que mi familiar puede obtener la atención médica que necesita sin tener que perjudicarme financieramente & 3,39 & 0,88 \\
\hline Tengo que gastar mucho más, por el cuidado médico que puedo pagar & 2,88 & 0,87 \\
\hline \multicolumn{3}{|l|}{ Dimensión tiempo dedicado por el médico } \\
\hline Muchas veces los médicos se apresuran demasiado cuando conversamos & 3,65 & 0,97 \\
\hline Las enfermeras generalmente están pendientes del cuidado de mi familiar & 3,87 & 0,83 \\
\hline \multicolumn{3}{|l|}{ Dimensión accesibilidad y conveniencia } \\
\hline Tengo fácil acceso a los médicos especialistas que necesita mi familiar & 3,61 & 0,80 \\
\hline Donde recibe atención médica, mi familiar tiene que esperar demasiado tiempo para recibir tratamiento & 3,07 & 1,07 \\
\hline Le resulta difícil de ver a su familiar fuera del horario de visita & 2,45 & 0,95 \\
\hline Puedo ser atendido por el médico cada vez que necesito o tenga un problema & 3,67 & 0,76 \\
\hline
\end{tabular}

dimensión satisfacción general obtuvo una media de 7,99 $\pm 1,09$, lo cual equivale a $79,90 \%$ de satisfacción, siendo así la dimensión con mayor puntuación favorable. Sin embargo, la dimensión más

afectada correspondió a forma interpersonal con una de media $6,05 \pm 0,62$, lo cual equivale a 60,50\%. La puntuación total obtuvo una valoración de 70,5\% de satisfacción. Tabla 5.

Tabla 5. Resultados de satisfacción del familiar por dimensiones, según la encuesta The Patient Satisfaction Questionnaire Short Form (PSQ-18) aplicada a familiares de pacientes hospitalizados en la Unidad de Cuidados Intensivos del Hospital Dos de Mayo, 2018.

\begin{tabular}{lccc} 
Dimensiones de satisfacción & Media & Desviación estándar & Satisfacción \% \\
Satisfacción general & 7,99 & 1,09 & 79,9 \\
\hline Calidad técnica & 14,98 & 2,19 & 74,9 \\
\hline Forma interpersonal & 6,05 & 0,62 & 60,5 \\
\hline Comunicación & 7,87 & 1,11 & 78,7 \\
\hline Aspectos financieros & 6,27 & 1,00 & 62,7 \\
\hline Tiempo dedicado por el médico & 7,49 & 1,35 & 74,9 \\
\hline Accesibilidad y conveniencia & 12,80 & 1,86 & 64,0 \\
\hline Puntuación total & 63,45 & 5,71 & 70,5 \\
\hline
\end{tabular}

\section{DISCUSIÓN}

Las familias requieren información relacionada con el plan de atención del paciente. Ante cualquier cambio y progreso general, es evidente que existe un marcado interés por conocer qué se esta haciendo por el paciente y por qué, ya que la información permite fundamentar a los familiares el posible pronóstico del paciente en forma razonable, disminuyendo así el estrés y la ansiedad. Un temor habitual de los familiares es que se pueda omitir información vital relacionada con la condición del paciente ${ }^{15}$.

La atención médica sigue siendo una de las actividades donde se centra la preocupación de los familiares. Los hallazgos del presente estudio evidenciaron resultados favorables al respecto, ya que el promedio de las puntuaciones 
tuvieron orientación positiva. Resultados similares fueron descritos por un estudio realizado en un hospital universitario por Holanda MS y col., donde $84,7 \%$ de los pacientes encuestados calificaron como muy buena su satisfacción con los cuidados brindados por el equipo médico, con énfasis en la experiencia profesional, las habilidades y competencias profesionales ${ }^{16}$. Uno de los resultados con puntuación promedio favorable correspondió a la dimensión calidad técnica; centrado en las condiciones necesarias que cuenta el área donde se encuentran los pacientes. Los resultados obtenidos podrían tener explicación en el hecho que las instalaciones y estructura física son relativamente nuevas en comparación con otras áreas del hospital, cuya antigüedad supera los 50 años. La satisfacción de los familiares respecto a la infraestructura y otros componentes como la ubicación, el acceso, las condiciones de la sala de espera, la sala de información, la limpieza y el orden, han sido valorados con un alto porcentaje de satisfacción (98,7\%), de modo similar lo señala un estudio realizado en Martos-Casado y col. ${ }^{17}$. Es importante señalar que aspectos como comodidad, presencia/ausencia de ruido, iluminación, privacidad y comodidad de la cama, son tomados en cuenta en diversos estudios. Sin embargo, algunas investigaciones han señalado que la falta de luz y la ausencia de relojes, provoca la falta de referencias temporales y alteraciones en el ritmo circadiano; el exceso de iluminación nocturna impide la secreción de melatonina, indispensable para inducir el sueño, lo que ocasiona quejas por parte de los pacientes ya que dificulta el sueño por la excesiva luz y ruido. Asimismo, una de las quejas más frecuentes que expresan los familiares se refiere a las salas de espera, a las que califican como lugares incómodos, poco agradables y poco estéticos, lo cual evidencia una contradicción con la imagen institucional de un hospital que busca hacer de la unidad de cuidados intensivos un lugar mas agradable ${ }^{18}$.

Respecto a los resultados correspondientes a la dimensión forma interpersonal, la atención personalizada que brinda el personal médico obtuvo un promedio menor a 2,5, lo cual podría considerarse desfavorable, debido a que el nivel de ocupación de la unidad es elevado en forma permanente, limitando la atención personalizada de los pacientes. Estos hallazgos reafirman la necesidad de hacer sentir que el equipo de profesionales se preocupa por el paciente y que reciba la mejor atención posible con énfasis en el tratamiento debido a la condición critica de los pacientes, necesidades de procedimientos médicos muy especializados, y constante observación y cuidados. Es importante señalar que el cuidado crítico holístico centrado en el paciente y la familia está destinado a satisfacer las necesidades tanto del paciente como de sus familiares $^{19,20}$

Por otra parte, la información sobre el motivo de los exámenes médicos y pruebas de ayuda diagnóstica, tienen una relación directa con el entendimiento por parte de los familiares y el paciente en caso se encuentre consciente, como consecuencia de ello se califica la satisfacción. Estudios realizados en áreas críticas han informado que la satisfacción puede llegar a 47,3\%, como los descritos por Matzumura y col. ${ }^{21}$. A pesar que el presente estudio se realizó en una institución pública, los resultados al respecto podrían considerarse favorables, ya que el promedio de las respuestas presentó una tendencia positiva hacia la satisfacción. Sin embargo, los profesionales asumen que los familiares no comprenden la información por estar sometidos a una situación de estrés; cabe señalar que en la unidad de cuidados intensivos se brinda prioridad a la realización de pruebas de ayuda diagnóstica antes que a la puntualidad o a la información previa ${ }^{17,22}$.

De igual modo, la atención de un paciente critico sigue siendo un problema para los familiares, debido que en la mayoría de los casos los pacientes no cuentan con un sistema de seguro que permita cubrir los diversos gastos para el cuidado y tratamiento; estos son elevados y, en la mayoría de los casos, difíciles de cubrir, ocasionando un problema financiero para los familiares $22,23,24$. En algunos casos, los hospitales brindan un sistema de asistencia social y de cobertura a través del seguro integral. Un estudio similar realizado en el Hospital Sirio Libanes ubicado en Sao Paulo, reportó efectos de los costos de la atención y su impacto en los trastornos emocionales de los familiares, lo que hace suponer que estos se sienten más agobiados y sometidos a estrés ${ }^{25}$. Si bien es cierto el estudio no permitió relacionar el impacto de los costos en el estado emocional de los familiares, confirmó la realización de un gasto adicional para el cuidado del paciente.

Con respecto al régimen de visitas, este se valora en función al acceso y el tiempo permitido; asimismo, el número o cantidad de familiares que pueden ingresar a la visita, de tal manera que el horario de visitas resulte adecuado para la mayoría de los familiares. Algunas investigaciones mencionan que los profesionales que laboran en la unidad de cuidados intensivos deben estar preparados para abrir las puertas de esta área, para permitir que los familiares se involucren en el cuidado del paciente ${ }^{26,27}$. A pesar que los resultados sobre el acceso a las visitas fuera de horario presentó un promedio desfavorable, parece obedecer a que la cantidad de personas que desea ingresar es desbordante en la mayoría de los casos y se ve reflejado en la insatisfacción, a pesar de los esfuerzos por implementar flexibilidad en los horarios de visitas.

El presente estudio tuvo algunas limitaciones propias del diseño investigación, tipo, tamaño relativamente pequeño de la muestra y el posible sesgo en la selección debido a los criterios de exclusión, como no incluir a los miembros de la familia que hablan quechua u otra lengua nativa. Debido a consideraciones éticas y la vulnerabilidad de la población del estudio, no se consideró apropiado usar recordatorios, ni fue éticamente aceptable preguntar sobre las razones para no participar. Finalmente, señalar que los posibles costos de la atención del paciente y los trastornos emocionales informados por los miembros de la familia después de sus experiencias, podría suponer que las personas más agobiadas eran incapaces de participar en el estudio.

En conclusión, el instumento The Patient Satisfaction Questionnaire Short Form es de fácil aplicación y aceptación. Se evidenció un predominio del género masculino, con una estancia $\leq 10$ días, más del $90 \%$ de los pacientes ingresó 
por primera vez a la unidad y $87,4 \%$ fue coberturado por el seguro integral. Las explicaciones que brindan los médicos sobre el motivo de exámenes médicos y los aspectos de equipamiento obtuvieron puntaciones favorables, mientras la atención personalizada y la dificultad por visitar al paciente fuera del horario, obtuvieron puntuaciones desfavorables. Asimismo, la dimensión satisfacción general evidenció el mejor porcentaje de satisfacción, mientras la dimensión forma interpersonal el menor, todas las dimensiones superaron el $60 \%$ de satisfacción y 70,5\% de los familiares manifestó su satisfacción con respecto a la atención de su familiar.

\section{REFERENCIAS BIBLIOGRÁFICAS}

1. Bailey JJ, Sabbagh M, Loiselle CG, Boileau J, McVey L. Supporting families in the ICU: a descriptive correlational study of informational support, anxiety, and satisfaction with care. Intensive Crit Care Nurs. 2010; 26(2):114-22. DOI: 10.1016/j. iccn.2009.12.006

2. Kentish-Barnes N, Lemiale V, Chaize M, Pochard F, Azoulay E. Assessing burden in families of critical care patients. Crit Care Med. 2009; 37(10 Suppl):S448-56. DOI: 10.1097/ CCM.0b013e3181b6e145

3. Lautrette A, Darmon M, Megarbane B, Joly LM, Chevret S, Adrie C, et al. A communication strategy and brochure for relatives of patients dying in the ICU. N Engl J Med. 2007; 356(5):469-78. DOI: 10.1056/NEJMoa063446

4. Jongerden IP, Slooter AJ, Peelen LM, Wessels H, Ram CM, Kesecioglu J, et al. Effect of intensive care environment on family and patient satisfaction: a before-after study. Intensive Care Med. 2013; 39(9):1626-34. DOI: 10.1007/s00134-013-2966-0

5. Kryworuchko J, Heyland DK. Using family satisfaction data to improve the processes of care in ICU. Intensive Care Med. 2009; 35(12):2015-7. DOI: 10.1007/s00134-009-1612-3

6. Al-Mutair AS, Plummer V, O'Brien A, Clerehan R. Family needs and involvement in the intensive care unit: a literature review. J Clin Nurs. 2013; 22(1314):1805-17. DOI: 10.1111/jocn.12065

7. Kodali S, Stametz RA, Bengier AC, Clarke DN, Layon AJ, Darer JD. Family experience with intensive care unit care: association of self- reported family conferences and family satisfaction. J Crit Care. 2014; 29(4):641-4. DOI: 10.1016/jrc.2014.03.012

8. Wallau RA, Guimarães HP, Falcão LFR, Lopes RD, Leal PHR, Senna APR, et al. Qualidade e humanização do atendimento em medicina intensiva. Qual a visão dos familiares? Rev Bras Ter Intensiva. 2006; 18(1):45-51. DOI: 10.1590/ S0103-507X2006000100009

9. Neves JL, Schwartz E, Echevarria- Guanilo ME, Amestoy SC, Mendieta MC, Lise F. Avaliação da satisfação de familiares de pacientes atendidos em unidades de terapia intensiva: revisão integrativa. Texto Contexto Enferm. 2018; 27(2):2-12. DOI: 10.1590/0104-070720180001800016

10. Rothen HU, Stricker KH, Heyland DK. Family satisfaction with critical care: measurements and messages. Curr Opin Crit Care. 2010; 16(6):62331. DOI: 10.1097/MCC.0b013e32833e9718

11. Stricker $\mathrm{KH}$, Kimberger O, Schmidlin K, Zwahlen $\mathrm{M}$, Mohr U, Rothen HU. Family satisfaction in the intensive care unit: what makes the difference? Intensive Care Met. 2009; 35(12):2051-9. DOI: 10.1007/s00134-009-1611-4

12. Chakraborty SN, Bhattacherjee S, Rahaman MA. A cross-sectional study on patient satisfaction in an urban health care centre of Siliguri Municipal Corporation, Darjeeling, West Bengal. Med J DY Patil Univ. 2016; 9(3):325-30. DOI: 10.4103/0975-2870.182502

13. Van den Broek JM, Brunsveld-Reinders $A H$, Zedlitz AM, Girbes AR, De Jonge, E, Arbous MS. Questionnaires on family satisfaction in the adult ICU: A systematic review including psychometric properties. Crit Care Med. 2015; 43(8):1731-44. DOI: $10.1097 / C C M .0000000000000980$

14. Marshall GN, Hays RD. The Patient Satisfaction Questionnaire Short Form (PSQ-18). Santa Monica, CA, EE.UU.: RAND; 1994.

15. Baharoon S, Al Yafi W, Al Qurashi A, Al Jadhali H, Tamim H, Alsafi E, et al. Family satisfaction in critical care units: Does an open visiting hours policy have an impact? J Patient Saf. 2017; 13(3):169-74. DOI: 10.1097/PTS.0000000000000140

16. Holanda MS, Ots E, Dominguez MJ, García A, Ruiz A, Castellanos A, et al. Medición de la satisfacción de los pacientes ingresados en unidad de cuidados intensivos y sus familiares. Med Intensiva. 2015; 39(1):4-12. DOI: 10.1016/j. medin.2013.12.008
17. Martos G, Aragón A, Gutiérrez N. Satisfacción de los familiares de los pacientes ingresados en una unidad de cuidados intensivos: percepción de los familiares y los profesionales. Enferm Intensiva. 2014; 25(4):123-72. DOI: 10.1016/j. enfi.2014.10.001

18. Escudero D, Viña L, Calleja C. Por una UCl de puertas abiertas, más confortable y humana. Es tiempo de cambio. Med Intensiva. 2014; 38(6):3715. DOI: 10.1016/j.medin.2014.01.005

19. Shorofi SA, Jannati Y, Moghaddam HR, YazdaniCarati J. Psychosocial needs of families of intensive care patients: perceptions of nurses and families. Niger Med J. 2016; 57(1):10-8. DOI: 10.4103/0300 1652.180557

20. Lolaty HA, Bagheri-Nesami M, Shorofi SA, Golzarodi T, Charati JY. The effects of family-friend visits on anxiety, physiological indices and wellbeing of $\mathrm{Ml}$ patients admitted to a coronary care unit. Complement Ther Clin Pract. 2014; 20(3):14751. DOI: 10.1016/j.ctcp.2014.03.002

21. Matzumura J, Gutiérrez H, Zamudio L. Satisfacción de la atención en el servicio de emergencia de una institución privada de salud. Qhalikay Revista de Ciencias de la Salud. 2018;2(1): 1-11.

22. Cehua E, Matzumura JP, Gutiérrez H, Zamudio L. Calidad de la información médica brindada a las pacientes atendidas en el servicio de emergencia de ginecología y obstetricia de un hospital categoria II-2 en Lima, Perú. REVCOG. 2017; 22(2):31-37.

23. Padilla-Fortunatti C, Rojas-Silva N, ArechabalaMantuliz MC. Análisis de la diferencia entre importancia y satisfacción de las necesidades de los familiares de pacientes críticos. Med Intensiva. 2018; 43(4):217-224. DOI:10.1016/j.medin.2018.06.010

24. Agärd AS, Egerod I, Tonnesen E, Lomborg K. From spouse to caregiver and back: a grounded theory study of post-intensive care unit spousal caregiving. J Adv Nurs. 2015; 71(8):1892-903. DOI: 10.1111/jan.12657

25. Fumis, RR, Ranzani OT, Martins PS, Schettino G. Emotional disorders in pairs of patients and their family members during and after ICU stay. PLoS One. 2015; 10(1):e0115332. DOI: 10.1371/journal. pone.0115332

26. Ríos-Briz N. Cuidados intensivos de puertas abiertas: ¿estamos preparados los profesionales? Enferm Intensiva. 2017; 28(4):187. DOI: 10.1016/j. enfi.2017. 04.004

27. Ayllón N, Montero P, Acebes MI, Sánchez J. Unidades de cuidados intensivos de puertas abiertas: perspectiva de los profesionales. Enferm Intensiva 2014; 25(2):72-7. DOI: 10.1016/j.enfi.2013.11.007 